\title{
Is there a role for pharmacogenetics in the dosing of fentanyl?
}

\author{
"At least, a part of this variation in pharmacokinetics and \\ pharmacodynamics is a consequence of a combination of, thus far, \\ poorly understood genetic factors."
}

First draft submitted: 29 January 2017; Accepted for publication: 29 January 2017; Published online: 27 March 2017

Keywords: fentanyl • pharmacogenetics • pharmacogenomics

Fentanyl is one of the most frequently used opioids for the treatment of severe chronic and acute cancer-related pain. It is the opioid of first choice in patients with renal impairment [1] and seems to be related with a lower incidence of severe constipation than other opioids [2]. Furthermore, it is available in various patient-friendly products for different clinical situations. The fentanyl patches result in continuous delivery of fentanyl during $72 \mathrm{~h}$ and are therefore ideal for the treatment of chronic severe pain. The formulations for buccal, sublingual or nasal delivery of fentanyl are, on the contrary, ideal for the management of acute exacerbations of pain, whereas the fentanyl solutions for intravenous or subcutaneous administration are ideal for tailored pain control in the clinic, for example, in the postoperative setting. Despite these, at first sight, practical fentanyl products, adequate pain relief without serious adverse effects is only reached in $75-90 \%$ of the patients $[3,4]$. Furthermore, large interindividual differences are found in the daily dosages needed to achieve pain control even in opioidnaive patients $[3,4]$. Some of this variation in response can be explained by pharmacokinetic variation, which also vary largely between individuals [5,6]. Only a relatively small part of this variation can be explained by known covariates like age, comedication, impaired liver function, BMI and heating of fentanyl patches (extensively reviewed by Kuip et al. [7]). Pharmacodynamic varia- tion caused by genotypes in receptor signaling or pain modulators, thereby effecting pain sensitivity and opioid-induced effects, may also play an important factor that is not completely unraveled [8]. These different mechanisms suggest that baseline pharmacogenetic screening before initiation of treatment may serve as a helpful diagnostic tool to predict the individual response to fentanyl.

\section{Pharmacokinetics}

Fentanyl is extensively metabolized by $N$-dealkylation to norfentanyl that is mediated by CYP3A4 and -3A5 (CYP3A). Less than $1 \%$ is metabolized by alkyl hydroxylation, $N$-dealkylation or amide hydrolysis to despropionylfentanyl, hydroxynorfentanyl and hydroxynorfentanyl. Drug interaction with CYP3A4 inhibitors resulted in mixed responses. Severe and even lethal fentanyl intoxications have been reported for fentanyl when given in combination with strong CYP3A inhibitors [9]. The effect of CYP3A inhibitors on the systemic exposure to fentanyl is however inconclusive. Co-administration with ritonavir, a very potent irreversible CYP3A inhibitor, resulted in almost twofold increase in exposure to fentanyl, whereas other potent reversible CYP3A inhibitors resulted in modest increases (26-39\%) or in case of itraconazole, no significant effect on fentanyl exposure [10-13].

Ziesenitz et al. also studied the metabolic clearance of fentanyl to norfentanyl when

\section{Stijn LW Koolen}

Department of Medical Oncology, Erasmus MC Cancer Institute, Rotterdam, The Netherlands

Carin CD Van der Rijt Author for correspondence: Department of Medical Oncology, Erasmus MC Cancer Institute, Rotterdam, The Netherlands and

Netherlands Comprehensive Cancer Organisation, The Netherlands

Tel.: +31 107041906

Fax: +31 107041003

c.vanderrijt@erasmusmc.nl 
given with or without ketoconazole [11]. Despite the modest increase in fentanyl exposure, ketoconazole resulted in almost complete inhibition of the fentanyl metabolic clearance. The high extraction rate of fentanyl might explain this discrepancy. Mainly hepatic blood flow determines total clearance instead of the intrinsic ability of the liver to metabolize a high extraction drug. Ritonavir on the other hand did result in an enormous increase in systemic exposure to fentanyl and changed fentanyl from a high extraction drug into a low extraction drug [13]. Unlike ketoconazole, ritonavir has numerous effects on other drug-metabolizing enzymes and transporters. Thus, possibly other enzymes play a role in fentanyl metabolism and genetic alterations in genes encoding for these enzymes have predictive value for fentanyl systemic exposure. SNPs in genes encoding for these metabolizing enzymes and transporters may thus influence fentanyl pharmacokinetics and thereby its analgesic effect.

\section{"...there is not a role yet for pharmacogenetics in the dosing of fentanyl."}

Indeed, different SNPs have been investigated in relation to fentanyl pharmacokinetics and treatment outcome $[7,8]$. Mixed results were found for the $\mathrm{CYP} A 5^{*} 3 \mathrm{SNP}$. This SNP results in reduced activity of the CYP3A5 enzyme and was linked with a twofold increase in systemic exposure to fentanyl [14]. Barratt et al., however, could not confirm the effect of the CYP3A genotypes in the large cross-sectional EPOS study [15]. They found that the CYP3A4*22 and CYP $3 A 5^{*} 3$ accounted for only a very small proportion $(<2 \%)$ of the metabolic ratio of norfentanyl:fentanyl and did not affect plasma levels of fentanyl. Other SNPs in genes encoding for CYP3A4 and the transporters ABCB1 and SCLCO1B1 were investigated in other studies but could not be related with exposure to fentanyl (reviewed in [7] and [8]).

\section{Pharmacodynamics}

Fentanyl exerts its effect via binding with the $\mu$-opioid receptor. It is $60-100$ times more potent than morphine, becauce it is highly lipophilic and therefore penetrates more easily the CNS [16]. Variability may be caused by differences in receptor affinity, due to genetic heterogeneity of the opioid receptors (OPRM1, OPRK1 and OPRD1) or variation in the modulating, inhibitory catecholamine neurotransmission, thereby affecting pain sensitivity and opioid effects [17]. Changes in catechol- $O$-methyltransferase (COMT) activity modifies adrenergic and serotonergic neurotransmission and thereby not only influence pain sen- sitivity and opioid requirements [8] but also influences opioid-induced central side effects including nausea and vomiting [17]. The effect sizes were, however, contradictory and were mostly related to morphine treatment. Based on four SNPs in the COMT gene associated with pain sensitivity, three pain sensitivity haplotypes were constructed [18]. These COMT haplotypes seemed to account better for variability in pain sensation [19]. However, this finding could not be confirmed in a recent study of our group in an exploratory analysis of 233 patients with cancer-related pain treated with fentanyl; in this group, treatment failure was not related to the COMT haplotypes [4].

Probably the most important gene in relation to fentanyl pharmacodynamics is the OPRM1 gene. This gene encodes for the $\mu$-opiod receptor. The A118 SNP is the most studied variant in the OPRM1 gene. The variant allele was associated with decreased effects of opioids. Clinically, this effect was modest and required only slightly higher opioid doses (extensively reviewed by Walter et al. [20]). Another enzyme closely related to the opioid receptor is $\beta$-arrestin. Binding to the opioid receptor by an agonist results in recruitment of $\beta$-arrestin, which promotes $\mu$-opioid receptor density. Polymorphisms in the gene encoding for $\beta$-arrestin, $A R R B 2$, were positively correlated with response to morphine but are not yet associated with response to fentanyl treatment. Variability in pharmacodynamics outcome of fentanyl and opioid treatment in general can probably not relevantly be explained by single SNPs. Future studies should therefore focus on genegene interactions and explore the prognostic value of multiple level interactions [21]. These analyses should not be limited by the most likely candidates but should also include enzymes associated with opioid side effects. For example, SNPs in the HTR3B gene which encodes for the 5-hydroxytryptamine $3 \mathrm{~b}$ receptor have been associated with opioid-induced nausea [22]. Furthermore, genetic variability in pro- and antiinflammatory cytokines, which may promote pain or induce analgesic effects, should also be taken into account [23].

\section{Conclusion}

With the available evidence of genetic factors in relation to fentanyl response, we must conclude that there is not a role yet for pharmacogenetics in the dosing of fentanyl. However, there is still a large variation in response that is only marginally explained with the known covariates. At least, a part of this variation in pharmacokinetics and pharmacodynamics is a consequence of a combination of, thus far, poorly understood genetic factors. This warrants further pharmacogenetic research in relation to fentanyl treatment, 
possibly resulting in pharmacogentic-guided dosing of fentanyl in the near future.

\section{Financial \& competing interests disclosure}

$C$ van der Rijt has received a grant from Prostrakan for an investigator initiated study on the pharmacokinetics of sublin-

\section{References}

1 Caraceni A, Hanks G, Kaasa S et al. Use of opioid analgesics in the treatment of cancer pain: evidence-based recommendations from the EAPC. Lancet Oncol. 13, e58-e68 (2012).

2 Tassinari D, Sartori S, Tamburini E et al. Adverse effects of transdermal opiates treating moderate-severe cancer pain in comparison to long-acting morphine: a meta-analysis and systematic review of the literature. J. Palliat. Med. 11, 492-501 (2008).

3 Mystakidou K, Parpa E, Tsilika E et al. Pain management of cancer patients with transdermal fentanyl: a study of 1828 step I, II, \& III transfers. J. Pain 5, 119-132 (2004).

4 Oosten AW, Matic M, van Schaik RH et al. Opioid treatment failure in cancer patients: the role of clinical and genetic factors. Pharmacogenomics 17, 1391-1403 (2016).

5 Oosten AW, Abrantes JA, Jönsson S et al. Treatment with subcutaneous and transdermal fentanyl: results from a population pharmacokinetic study in cancer patients. Eur. J. Clin. Pharmacol. 72, 459-467 (2016).

6 Fung DL, Eisele JH. Fentanyl pharmacokinetics in awake volunteers. J. Clin. Pharmacol. 20, 652-658 (1980).

7 Kuip EJ, Zandvliet ML, Koolen SL, Mathijssen RH, van der Rijt CC. A review of factors explaining variability in fentanyl pharmacokinetics; focus on implications for cancer patients. Br. J. Clin. Pharmacol. 83(2), 294-313 (2017).

8 Nielsen LM, Olesen AE, Branford R, Christrup LL, Sato $\mathrm{H}$, Drewes AM. Association between human pain-related genotypes and variability in opioid analgesia: an updated review. Pain Pract. 15, 580-594 (2015).

9 Horton R, Barber C. Opioid-induced respiratory depression resulting from transdermal fentanyl-clarithromycin drug interaction in a patient with advanced COPD. J. Pain Symptom Manage. 37, e2-e5 (2009).

10 Palkama VJ, Neuvonen PJ, Olkkola KT. The CYP 3A4 inhibitor itraconazole has no effect on the pharmacokinetics of i.v. fentanyl. Br. J. Anaesth. 81, 598-600 (1998).

11 Ziesenitz VC, Konig SK, Mahlke NS, Skopp G, Haefeli WE, Mikus G. Pharmacokinetic interaction of intravenous fentanyl with ketoconazole. J. Clin. Pharmacol. 55, 708-717 (2015).

12 Saari TI, Laine K, Neuvonen M, Neuvonen PJ, Olkkola $\mathrm{KT}$. Effect of voriconazole and fluconazole on the gual fentanyl. The authors have no other financial involvement with any organization or entity with a financial interest in or financial conflict with the subject matter or materials discussed in the manuscript apart from those disclosed.

No writing assistance was utilized in the production of this manuscript.

pharmacokinetics of intravenous fentanyl. Eur. J. Clin. Pharmacol. 64, 25-30 (2008).

13 Olkkola KT, Palkama VJ, Neuvonen PJ. Ritonavir's role in reducing fentanyl clearance and prolonging its half-life. Anesthesiology 91, 681-685 (1999).

14 Takashina Y, Naito T, Mino Y, Yagi T, Ohnishi K, Kawakami J. Impact of $C Y P 3 A 5$ and $A B C B 1$ gene polymorphisms on fentanyl pharmacokinetics and clinical responses in cancer patients undergoing conversion to a transdermal system. Drug Metab. Pharmacokinet. 27, 414-421 (2012).

15 Barratt DT, Bandak B, Klepstad P et al. Genetic, pathological and physiological determinants of transdermal fentanyl pharmacokinetics in 620 cancer patients of the EPOS study. Pharmacogenet. Genomics 24, 185-194 (2014).

16 Donner B, Zenz M, Tryba M, Strumpf M. Direct conversion from oral morphine to transdermal fentanyl: a multicenter study in patients with cancer pain. Pain 64, 527-534 (1996).

17 Ross JR, Riley J, Taegetmeyer AB et al. Genetic variation and response to morphine in cancer patients: catechol- $O$ -methyltransferase and multidrug resistance-1 gene polymorphisms are associated with central side effects. Cancer 112, 1390-1403 (2008).

18 Diatchenko L, Nackley AG, Slade GD et al. Catechol-Omethyltransferase gene polymorphisms are associated with multiple pain-evoking stimuli. Pain 125, 216-224 (2006).

19 Zhang F, TongJ, Hu J et al. COMT gene haplotypes are closely associated with postoperative fentanyl dose in patients. Anesth. Analg. 120, 933-940 (2015).

20 Walter C, Doehring A, Oertel BG, Lotsch J. micro-opioid receptor gene variant $O P R M 1118 \mathrm{~A}>\mathrm{G}$ : a summary of its molecular and clinical consequences for pain. Pharmacogenomics 14, 1915-1925 (2013).

21 Branford R, Droney J, Ross JR. Opioid genetics: the key to personalized pain control? Clin. Genet. 82, 301-310 (2012).

22 Laugsand EA, Fladvad T, Skorpen F et al. Clinical and genetic factors associated with nausea and vomiting in cancer patients receiving opioids. Eur. J. Cancer 47, 1682-1691 (2011).

23 Uceyler N, Eberle T, Rolke R, Birklein F, Sommer C. Differential expression patterns of cytokines in complex regional pain syndrome. Pain 132, 195-205 (2007). 